\title{
Pain treatment with high-dose, controlled-release oxycodone: an Italian perspective
}

\author{
Fabio Ferrarese \\ Gioia Becchimanzi² \\ Massimo Bernardo 3 \\ Maria Anna Conte ${ }^{4}$ \\ Angela Gioia ${ }^{5}$ \\ Davide Ottaviani ${ }^{6}$ \\ Rosa Palomba ${ }^{7}$ \\ Gianfranco Pedelini ${ }^{8}$ \\ Laura Rigotti ${ }^{9}$ \\ Elvira Scelzi ${ }^{10}$ \\ 'Ospedale Ca'Foncello, Treviso; ${ }^{2}$ Cure \\ Palliative, Terapia Antalgica, Ospedale \\ Civile Pinerolo, Torino; ${ }^{3}$ Serv. Cure \\ Palliative, Osp. Bolzano, Bolzano; \\ ${ }^{4}$ Casa di cura "Pineta del Carso", \\ Aurisina, Trieste; ${ }^{5}$ Terapia Antalgica, \\ Ospedale S. Chiara, Pisa; ${ }^{6}$ Ospedale \\ Molinette, Torino; ${ }^{7} \mathrm{AOU}$ Federico \\ II, Napoli; ${ }^{8}$ Hospice S. Cristoforo \\ Cologna Veneta, Verona; ${ }^{9}$ Servizio \\ Cure Palliative Rovereto, Trento; \\ 10 U.O. di Oncologia Castelfranco \\ Veneto, Treviso, Italy
}

Objective: To investigate the possible role and tolerability of high-dose $(>160 \mathrm{mg} /$ day $)$ oxycodone controlled release (CR) for the treatment of cancer and non-cancer pain.

Design: 227 patients with cancer or non-cancer pain were enrolled in an open-label, multi-center, Italian study in order to assess the adequacy of their existing pain management (using a numerical rating scale $[\mathrm{NRS}]$ ) and the possible benefit high-dose oxycodone CR may offer patients experiencing uncontrolled pain.

Results: Pain was poorly controlled at baseline, with only $18.1 \%$ of patients reporting adequate pain relief (NRS $<3.5$ ). All other patients reported uncontrolled pain, with an average NRS of 7.81. At baseline assessment, $47.89 \%$ of patients had been in pain for up to 3 months, $32.82 \%$ for 3-6 months, and $19.19 \%$ for more than 6 months. After baseline assessment, patients were switched to oxycodone CR monotherapy. The starting dose was individualized to each patient and titrated up over a 3- to 4-day period until effective pain management was achieved. Treatment was continued for an average of 37.24 days during the study. Pain control (final mean NRS of 2.85) was attained with an average dose of oxycodone CR $221.84 \mathrm{mg} /$ day. Standard adverse events (including constipations, nausea, and vomiting) were recorded in $39.64 \%$ of patients receiving high-dose oxycodone $\mathrm{CR}$ monotherapy. Side-effects tended to subside after the initial week of treatment and did not result in any participants leaving the study.

Conclusion: High-dose oxycodone $\mathrm{CR}$ can achieve rapid and effective management of moderate to severe cancer and non-cancer pain with minimum side-effects.

Keywords: oxycodone CR, pain, high-dose, opioid, moderate-severe-pain, cancer, noncancer

\section{Introduction}

Living with pain is a reality that affects millions of people worldwide. Cancer pain alone is a significant issue, affecting an estimated 17 million people every year (Goudas et al 2005). Evidence suggests that around $70 \%$ of patients living with cancer have to endure chronic pain: $70 \%$ of which is caused by the disease, $20 \%$ by therapy, and $10 \%$ by other factors (Italian Department of Health 2007). Non-cancer-related pain also affects a significant number of people worldwide (Furlan et al 2006) and is thought to affect over a third of the population in developed countries. Data from the International Association for the Study of Pain (IASP) indicate that chronic pain is more common among women, affecting $39.6 \%$ of women compared with a prevalence of $31.0 \%$ among men (Yang et al 2007).

It has been shown on a pan-European level that, in addition to its impact on the quality of life (QoL) of affected individuals and their families, chronic pain also has a significant social and economic burden. Persistent pain can affect a patient's ability to perform simple tasks which, combined with a lack of availability of treatments and effective therapies, can undermine their ability to work. This is not only valid at a European level, but also holds true on a national scale. In Italy, $83.2 \%$ of cancer patients were found to be in pain as a result of their condition, and, although 
$97 \%$ of them were receiving therapy, the treatment was not appropriately tailored to relieve the intensity of pain experienced (Costantini et al 2005).

Pain is often the main presenting symptom when patients visit their primary care physician. The location and nature of the pain can inform doctors on the cause and possible pathology of a patient's condition. In Italy, however, diagnosis and subsequent treatment choice are often inadequate, with a significant $42 \%$ of cancer patients describing their pain as 'intolerable' despite treatment (Italian Medical Oncology Association 2007).

The European Pain in Cancer (EPIC) Survey findings provided further insight into the nature and prevalence of cancer pain at presentation (European Pain in Cancer Survey 2007). The survey encompassed 12 European countries and involved a total of 4824 patients, of whom 457 were Italian. Across Europe $73 \%$ of patients reported pain associated with their cancer, with approximately one in three (33\%) identifying it as the main symptom of their condition. This number was higher for Italy, where almost all Italian participants (95\%) reported pain associated with their cancer, and $42 \%$ identified pain as their key symptom.

More than half $(56 \%)$ of patients reported pain at the time of questioning, while $96 \%$ of patients in the survey experienced pain at least once a month, and 75\% weekly. At the time of the survey, nearly $47 \%$ of patients had been suffering pain for at least 3 months, and $6 \%$ reported having been in constant pain for more than a year.

Patients were asked to indicate the severity of their pain on a numerical rating scale (NRS) that ran from 1 to 10 , with 10 indicating the worst pain possible. More than $96 \%$ of patients classified their pain as moderate-to-severe, with an NRS of $>5$. Although $98 \%$ of patients involved in the study had received some form of pain treatment, only $24 \%$ of pan-European participants considered their treatment effective. This number was even lower for the Italian participants, among whom only $16 \%$ felt their treatment was effective. A significant $70 \%$ of patients involved in the study reported a belief that effective pharmacological pain control was not possible (European Pain in Cancer Survey 2007).

Poor pain control, as recorded in the study, contributes to the psychosocial and physical effects associated with chronic pain. In addition to the physical sensation of pain, patients can also experience sleep disturbance, fear, anxiety, depression, weight loss, reduced physical activity, social isolation, and problems with social interaction that often result in problems finding and maintaining employment. Chronic pain's high prevalence and debilitating nature result in a significant combined economic and social burden.

The impact of chronic pain on the patient, society, and the economy highlights the need to focus on, and address, pain on both a national and international scale. Recognition of the importance of finding an efficacious approach to pain management leads to arguments supporting the extensive use of opioids in chronic pain management (Ballantyne 2007). Opioids are considered by some to be integral to effective pain management, and key to minimizing the suffering associated with persistent pain (European Pain in Cancer Survey 2007), its deleterious physical effects (Carr et al 1999; Kehlet et al 2003; American Academy of Pain Medicine Council on Ethics 2005), and its erosion of a patient's autonomy, dignity, and decision-making capacity. However, the potentially addictive nature of opioids is also recognized, and caution is advised to ensure that the 'principle of balance' is upheld. The principle of balance maintains that efforts to address abuse of therapy may be necessary, but they should not interfere with legitimate medical practice and patient care (Brennen et al 2005; Dubois 2005). Furthermore, long-term studies into the use of opioids have not only found that prolonged treatment can improve patients' functioning and QoL, but also that it is possible to reach, and maintain, a stable (non-escalating) effective dose. The established side-effects associated with opioid use, such as nausea, sedation, constipation, and itching, also tend to subside with prolonged use, as side-effect tolerance appears to develop without an associated abrogation of analgesic effect.

The opioid oxycodone controlled release (CR) is commonly used for pain relief in cancer patients, and may be considered for first-line oral therapy. In a study on visceral pain, Staahl et al (2007) demonstrated that oxycodone $\mathrm{CR}$ has superior analgesia to morphine. In addition, the European Federation of Neurological Societies' (EFNS) recently recommended use of oxycodone alone for the treatment of painful polyneuropathy (PPN) at a level rating of $\mathrm{A}$ (that requiring the existence of a strong evidence base) (Attal et al 2006). A recent study investigating the efficacy and tolerability of high-dose oxycodone $\mathrm{CR}$ achieved optimum pain control at a dosage of oxycodone CR $224 \mathrm{mg}$ /day; resulting in a significant reduction in pain intensity (final NRS $<3$ ) with a favorable side-effect profile (Mameli et al 2006).

Yet, published evidence on the use of high-dose oxycodone $\mathrm{CR}$ in patients with terminal cancer remains limited (Bercovitch et al 2006). This study sought to address the gap in the evidence base by assessing the possible role and tolerability of high-dose ( $>160 \mathrm{mg} /$ day) oxycodone $\mathrm{CR}$ in the management of cancer and non-cancer pain. 


\section{Methods}

The study was designed as an open-label, multi-center, observational trial carried out across 10 locations. It ran for 3 months (from 1 April to 30 June 2007) and involved a total of 227 consecutive patients: 207 patients with cancer pain and 20 patients with non-cancer pain. A baseline pain evaluation was carried out using a NRS, and previous treatments were recorded to establish the most commonly prescribed therapy, and then correlated against the patient's perception of treatment efficacy.

The goal of the study was to reduce the intensity of pain experienced by participants. The study sought to establish whether prescription of an appropriate therapy, as recommended by the World Health Organization's (WHO) guiding principles: 'by the clock, by the mouth, by the ladder', could improve patients' pain relief (WHO 1996).

Patients included in the study were over 18 years of age, had a baseline NRS score of $>4$ and were able to take oral medication. Patients were excluded from the study if they were undergoing current radiotherapy treatment, required modification of adjuvant treatments, or were known to be intolerant to oxycodone.

Baseline assessments included cause of pain, duration of pain experienced prior to study, history of pain relief prescribed, treatment setting, and intensity of pain felt (indicative of treatment efficacy) using a NRS. After baseline assessment, patients were switched to oxycodone CR monotherapy and monitored for at least 21 days. The change in therapy followed recommendations outlined in Doyle et al (1997). Existing therapy was halted completely as study patients reported uncontrolled pain on current treatment (NRS $>4$ ) and were then switched to oxycodone $\mathrm{CR}$. After the change from previous treatment to oxycodone $\mathrm{CR}$, patients' pain score was evaluated and the high-dose oxycodone CR dosage was titrated up until adequate control (NRS $\leq 2.9$ ) was achieved.

Duration of therapy varied depending on date of enrolment in the study, but high-dose oxycodone monotherapy was taken by study participants for an average of 37.24 days. Oxycodone CR was chosen as the reference drug because of its pharmacokinetics, pharmacodynamics, and demonstrable efficacy in both cancer and non-cancer pain. The setting for treatment administration was not altered for the study. Initial dose was individualized to each patient, using standard conversion tables (Doyle et al 1997) and consideration of pharmacological history (previous treatment and dosage used), and titrated up (over a 3- to 4-day period) until effective pain control was achieved.

\section{Results}

During the 3-month study period, 227 patients from 10 sites were evaluated. The patient population comprised $40 \%$ women $(\mathrm{n}=90)$ and $60 \%$ male $(\mathrm{n}=137)$. Patients were aged $27-84$ years (average 63.76 years). Within the $91.18 \%$ $(n=207)$ of patients with cancer, the following categories were defined: $23.34 \%(n=53)$ lung; $13.21 \%(n=30)$ breast; $10.13 \%(\mathrm{n}=23)$ prostate; $9.25 \%(\mathrm{n}=21)$ pancreas; and $6.16 \%(\mathrm{n}=14)$ colon (Figure 1; Table 1).

Study participants were administered treatment in a number of different settings: outpatient $(\mathrm{n}=88)$; in the home

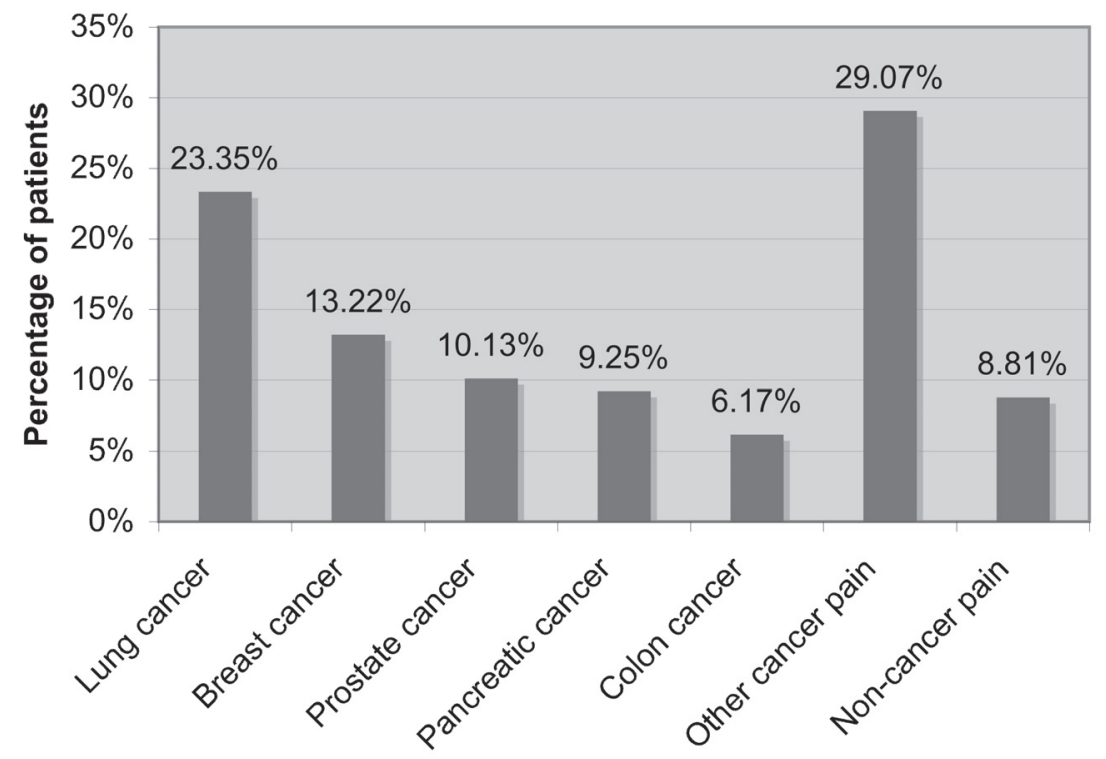

Figure I Cause of pain among study participants at baseline. 
Table I Summary of patient demographics at baseline $(n=227)$

\begin{tabular}{|c|c|c|c|c|c|}
\hline Sex & Female & Male & & & \\
\hline & $90(40 \%)$ & 137 (60\%) & & & \\
\hline \multirow[t]{2}{*}{ Age } & Range (years) & Average (years) & & & \\
\hline & $27-84$ & 63.76 & & & \\
\hline Cancer pain & lung & breast & prostate & pancreas & colon \\
\hline 207 (9l.18\%) & 53 (23.34\%) & 30 (I3.2I\%) & $23(10.13 \%)$ & 21 (9.25\%) & $14(6.16 \%)$ \\
\hline
\end{tabular}

( $n=64)$; hospice $(n=33)$; secondary care hospital $(n=24)$; day hospital $(n=17)$; and residential service agency $(n=1)$ (see Figure 2).

Of the 198 participants who responded to questioning at baseline, $47.98 \%(n=95)$ had been in pain for $\leq 3$ months, $32.83 \%(n=65)$ for $3-6$ months, and $19.19 \%(n=38)$ for $\geq 6$ months (Figure 3).

Evaluation of pain intensity was also assessed at study outset. The overall NRS calculated for participants at the outset of the study was 7.73, indicating that pain was uncontrolled among most patients. Baseline analysis reported controlled pain (defined as NRS 2.9, $\mathrm{p}<0.00001$ ) in $8.1 \%$ $(n=41)$ of patients, all of whom were receiving high-dose oxycodone CR (an average dose of $241 \mathrm{mg} /$ day). All other patients reported uncontrolled pain, with an average NRS of 7.81. Of note, $3.96 \%(n=9)$ recorded an NRS of 5 , while a significant $24.67 \%(\mathrm{n}=56)$ indicated a NRS of 8 , and $10.13 \%(n=23)$ recorded a maximum 10 on the NRS (Figure 4; Table 2).

A range of pharmacological agents were in use among patients who reported uncontrolled pain (Figure 5): transdermal fentanyl (30.0\%); morphine (12.8\%); transdermal buprenorphine (5.3\%); weak opioids (6.2\%); and non-steroidal anti-inflammatory drugs (NSAIDs) (1.3\%). Of patients who reported poor pain control, $42.2 \%$ were treated with low-dose ( $<80 \mathrm{mg}$ /day) oxycodone (either oxycodone/acetaminophen or oxycodone CR). Analysis found that breakthrough pain had been treated with immediate-release morphine (33.02\%), transmucosal fentanyl (24.18\%), NSAIDs (12.09\%), immediate-release oxycodone (in the form of oxycodone/acetaminophen) $(4.65 \%)$, and weak opioids $(0.9 \%)$.

During the study, all patients experiencing uncontrolled pain were switched from their original treatment to oxycodone CR monotherapy. The initial dose administered was titrated up until effective pain relief was achieved. Pain control (NRS $\leq 2.9)$ was attained using an average dose of oxycodone CR $221.84 \mathrm{mg} /$ day, resulting in an average NRS of 2.85 at study end $(\mathrm{p}<0.00001)$. All patients on oxycodone $\mathrm{CR}$ in the study were treated for an average treatment duration of 37.24 days. Side-effects (including constipation, nausea, and vomiting) were recorded among $39.64 \%$ of patients on high-dose oxycodone CR monotherapy, but tended to subside

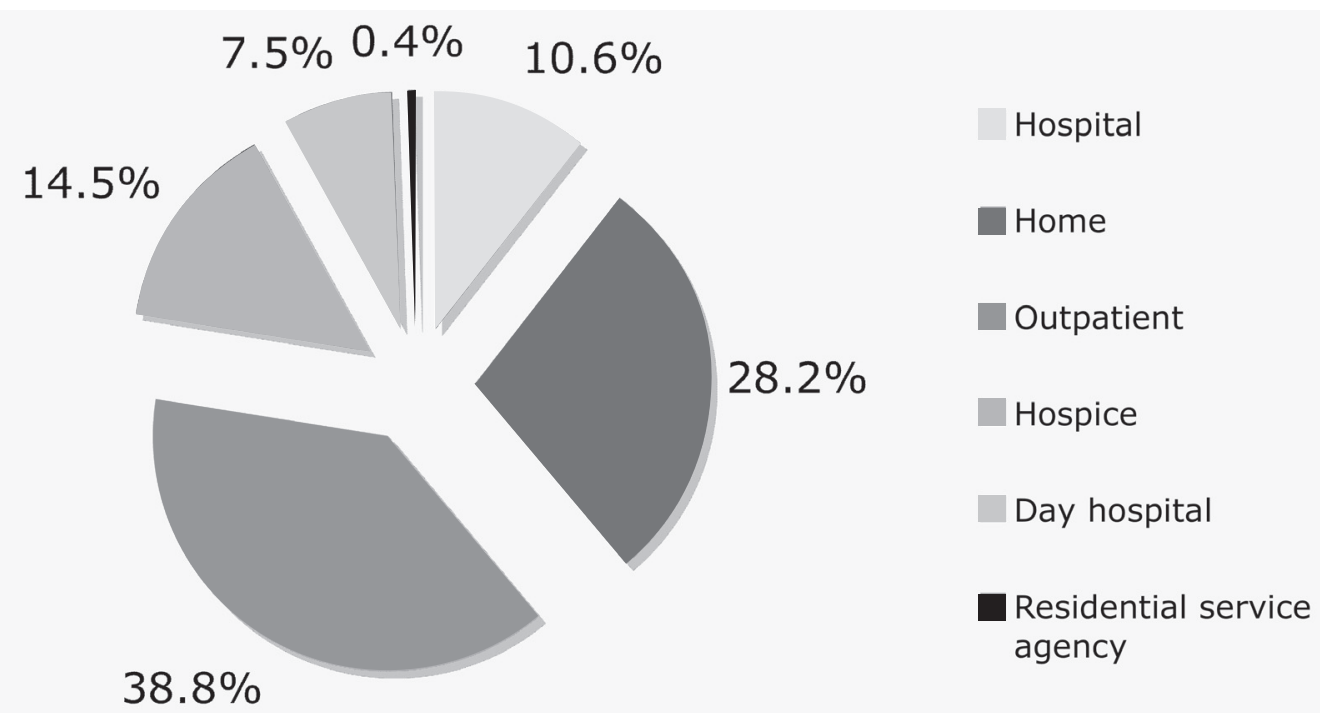

Figure 2 Setting for treatment administration. 


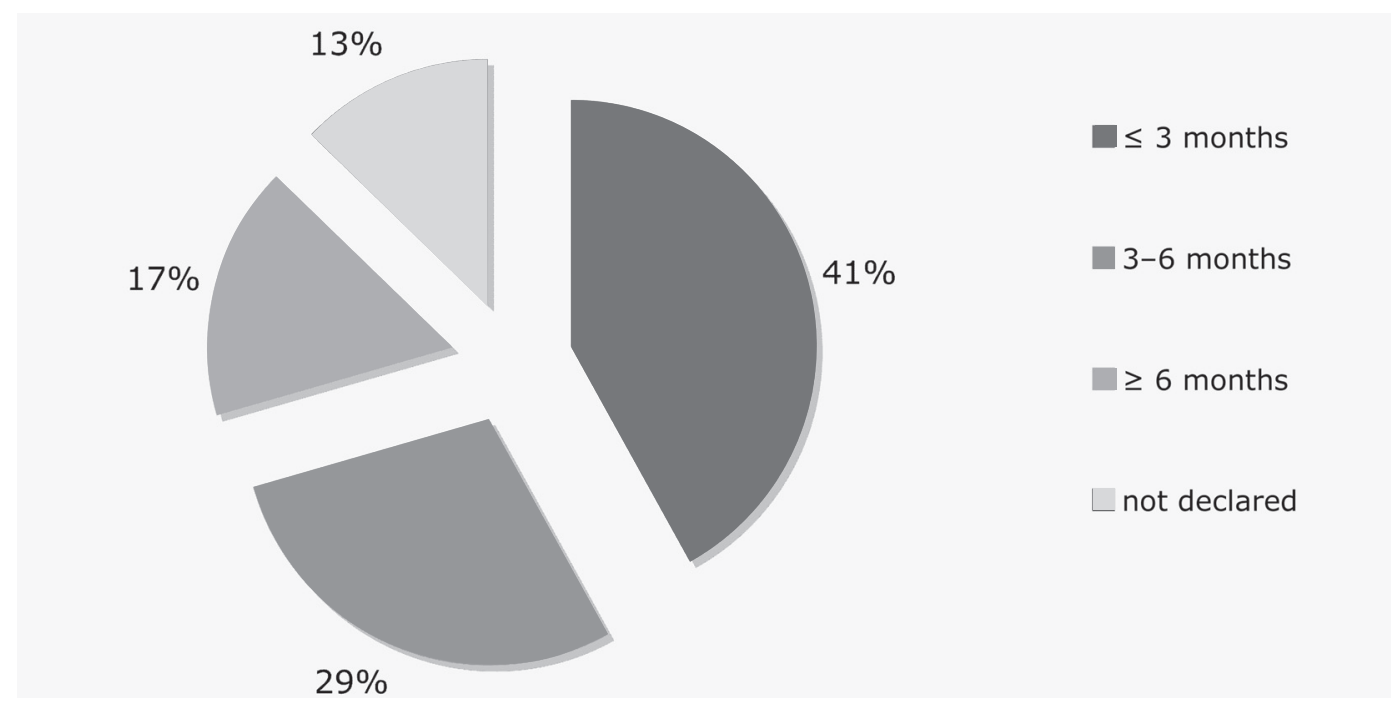

Figure 3 Duration of uncontrolled pain (in 3-month periods) among study participants at baseline.

after the first week of treatment and did not result in any patients leaving the study (Table 3).

\section{Discussion}

This study corroborates the poor management of both cancer and non-cancer pain in Italian patients. In a significant number of cases the wrong therapeutic approach was taken: transdermal treatments were used over oral agents for first-line therapy in more than a third of patients $(35.3 \%)$, despite no prior stabilization of their pain. In addition, the dose prescribed was not optimized in a large number of cases, as highlighted by the use of low-dose oxycodone in $42.2 \%$ of patients who reported persistent pain despite treatment. The study demonstrates that oxycodone CR can be used in such patients to achieve rapid control of moderate to severe pain, with minimal side-effects.

Although the mean duration of therapy in this trial was only 37.24 days, a separate study carried out over a 3-year period demonstrated that oxycodone $\mathrm{CR}$ can achieve adequate, longer-term pain management of non-cancer pain, without requiring a further increase in dose (Portenoy et al 2007).

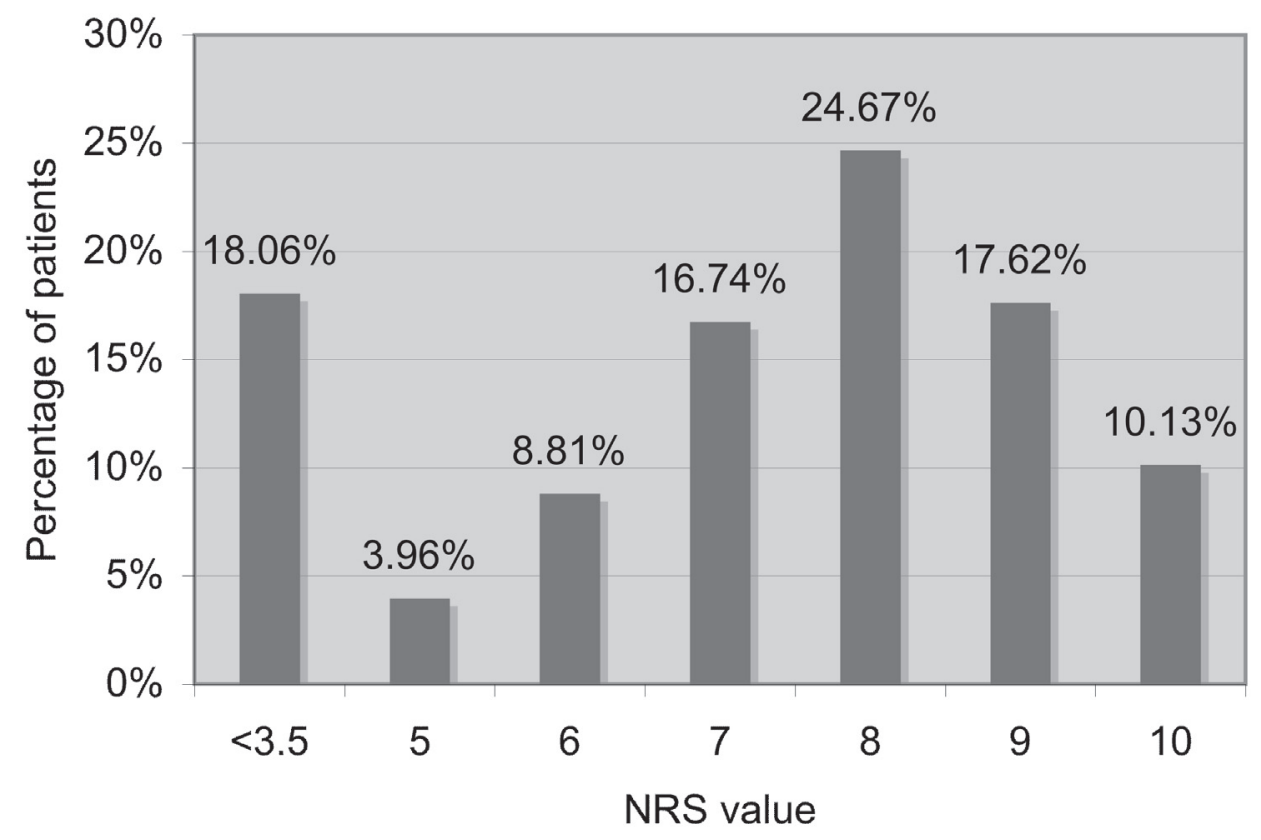

Figure 4 Pain severity as classified by study participants at baseline using numerical rating scale (NRS). 
Table 2 Summary of pain control at baseline $(n=198)$

\section{Average NRS score}

7.73

NRS 2.9 (controlled pain)

NRS 5 (uncontrolled pain)

NRS 8 (uncontrolled pain)

NRS 10 (uncontrolled pain)

Pain is one of the most common symptoms associated with cancer. It becomes more frequent as the cancer progresses, but may also be present during the early stages of the disease. Pain associated with cancer is largely due to the condition itself, but can (in $20 \%$ of cases) be caused by treatment complications.

An overlap in pain caused by antineoplastic treatments and that caused by cancer was recorded in around $10 \%$ of patients in a recent study $(\mathrm{n}=1095)$ by Caraceni et al (1999). Sources of cancer pain in the study were classified as bone lesion (due to metastases or to tumor invasion of bone and joint structures) in $41 \%$ of patients, neoplastic visceral damage in $28 \%$, and lesions of the nervous system in $28 \%$ of cases. These were further classified into the following pain pathophysiologies: somatic, visceral, and neuropathic. The most common pathophysiology was somatic-nociceptive pain $(32.0 \%)$, followed by somatic-neuropathic $(23.0 \%)$, pure visceral pain $(15.0 \%)$, pure neuropathic pain $(7.7 \%)$, and other syndromes of mixed pathophysiologies.

The aim of treatment in patients with advanced-stage cancer is improved QoL, which requires both efficacious treatment and adequate pain relief. Clinical experience confirms that optimal palliative care in cancer is associated with maximal pain relief. Yet, despite international guidelines on cancerpain management (WHO 1996) and availability of treatments that are effective in approximately $70 \%-90 \%$ of cases, up to $40 \%$ of cancer patients in Italy remain under-treated.

The European Society for Medical Oncology (ESMO) advocates the use of opioids as first-line therapy in moderate to severe cancer pain (ESMO Guidelines Task Force 2005, 2007). Guidance suggests that strong opioids can be safely administered, in increasing amounts, until pain relief is achieved. No ceiling dose is outlined, and no standard initial dose is suggested; the correct dose is one that achieves effective pain relief, and may vary among patients. The WHO recommends that pharmacological pain management should be guided by the core principles of: 'by the mouth, by the clock, by the ladder' (WHO 1996). 'By mouth' embodies the recommendation of oral therapy use (where possible) as it can be rapidly modified to changing intensities of pain. Transdermal formulations are recommended only in patients in whom pain management is stable, not as first-line therapy.

Oxycodone is derived from thebaine, an opium alkaloid. It is a semi-synthetic, pure opioid receptor agonist analgesic drug that appears to work by stimulating the $\mu$-opioid receptors found in the central nervous system (Glare et al 1993; Reder et al 1993; Citron et al 1998; Napp Pharmaceuticals 2007). Oxycodone is metabolized by the liver (by the cytochrome P450 2D6) to form oxymorphone and noroxycodone, which has only weak affinity for $\mu$-opioid receptors. Despite it being a potent analgesic, oxymorphone accounts for only $10 \%$ of oxycodone metabolites. Oxycodone, rather than

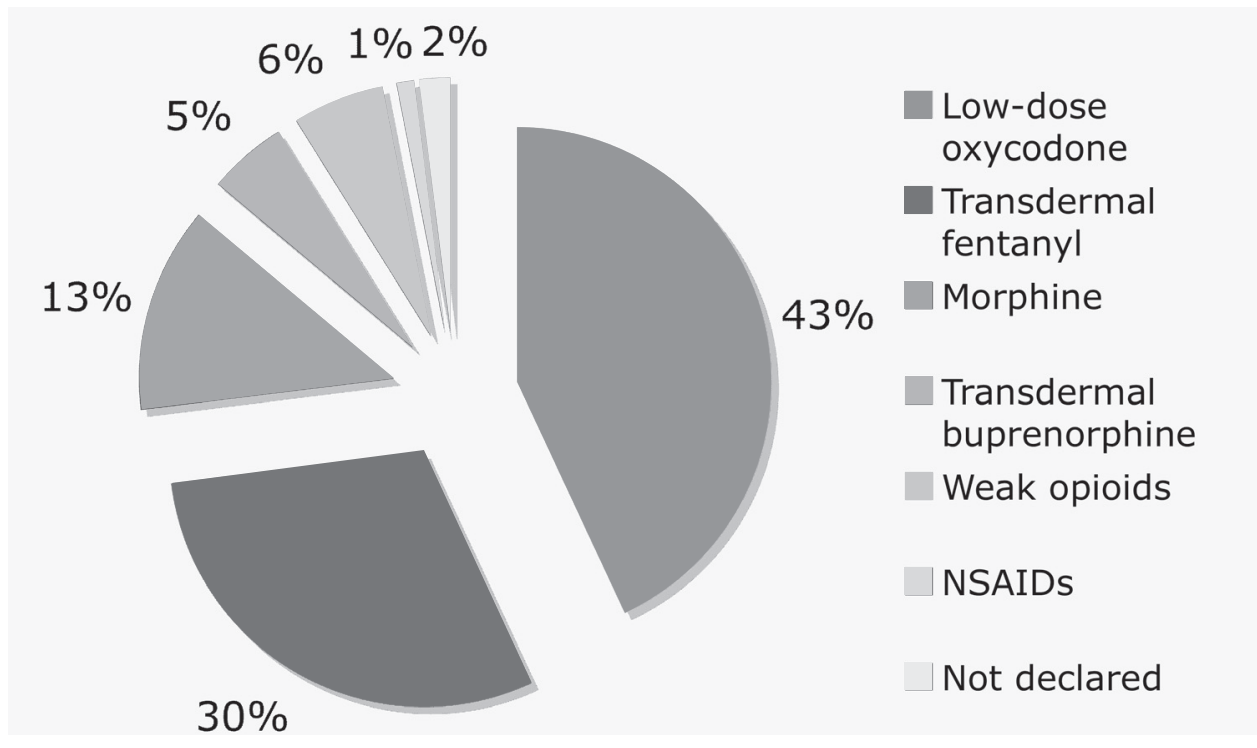

Figure 5 Distribution of pharmacological therapy that failed to achieve pain control at baseline. 
Table 3 Summary of study findings after patients' switch from previous pain mediction to high-dose oxycodone CR

\begin{tabular}{lllll}
\hline $\begin{array}{l}\text { Number of patients } \\
\text { evaluated }(\mathbf{N})\end{array}$ & $\begin{array}{l}\text { Average } \\
\text { oxycodone CR dose }\end{array}$ & $\begin{array}{l}\text { Average NRS } \\
\text { achieved }\end{array}$ & $\begin{array}{l}\text { Average treatment } \\
\text { duration (days) }\end{array}$ & $\begin{array}{l}\text { Percentage of patients } \\
\text { reporting side effects } \\
\text { (eg, constipation, } \\
\text { nausea, vomiting) }\end{array}$ \\
\hline 198 & & 2.85 & 37.24 & $39.64 \%$ \\
\hline
\end{tabular}

oxymorphone, appears to be responsible for both analgesia and side-effects. Recent studies have demonstrated a role for oxycodone as first-line pain therapy in place of morphine (Riley et al 2006), and evidence for its use, in both cancer and non-cancer pain, is growing.

\section{Conclusion}

Oxycodone $\mathrm{CR}$ is an oral therapeutic option approved for the treatment of moderate to severe pain. It has a similar safety and efficacy profile to morphine, but offers several therapeutic advantages. The results of its use at high dosages for pain relief in cancer and non-cancer patients demonstrate that an adequate dose can bring previously uncontrolled moderate to severe pain under control rapidly, with minimal side-effects. However, further randomized, controlled studies involving oxycodone $\mathrm{CR}$ and a control arm are required to increase evidence for the efficacy of oxycodone CR use in cancer and non-cancer pain.

\section{Acknowledgments}

The authors would like to thank Dr Berenato, Dr Cianfanelli, Dr Iodice, Dr Liotta and Dr Mazzocchi for their invaluable co-operation in this study and the editorial assistance of Alison Chisholm of Horizon Medical Publishing.

\section{Disclosures}

None of the authors has any conflicts of interest to disclose.

\section{References}

American Academy of Pain Medicine (AAPM) Council on Ethics. 2005. Ethics charter from American Academy of Pain Medicine. Pain Med, 6:203-12.

Attal N, Cruccu G, Haanpaa M, et al.; EFNS Task Force. 2006. EFNS guidelines on pharmacological treatment of neuropathic pain. Eur J Neurol, 13:1153-69.

Ballantyne JC. 2007. Opioid Analgesia: Perspectives on Right Use and Utility. Pain Physician, 10:479-91.

Bercovitch M, Adunsky A. 2006. High dose controlled-release oxycodone in hospice care. J Pain Palliat Care Pharmacother, 20:33-9.

Brennan TJ, Kehlet H. 2005. Preventive analgesia to reduce wound hyperalgesia and persistent postsurgical pain. Not an easy path. Anesthesiol, 103:681-3.

Caraceni A, Portenoy RK. 1999. An international survey of cancer pain characteristics and syndromes. International Association for the Study of Pain (IASP) Task Force on Cancer Pain. Pain, 82:263-74.
Carr DB, Goudas LC.1999. Acute pain. Lancet, 353:2051-8.

Citron ML, Kaplan R, Parris WC, et al. 1998. Long-term administration of controlled-release oxycodone tablets for the treatment of cancer pain. Cancer Invest, 16:562-71.

Costantini M, Beccaro M, Merlo F; ISDOC Study Group. 2005. The last three months of life of Italian cancer patients. Methods, sample characteristics and response rate of the Italian Survey of the Dying of Cancer (ISDOC). Palliat Med, 19:628-38.

Doyle D, Hanks GWC, MacDonald N. 1997. Modified Oxford Textbook of Palliative Medicine (2nd ed). Oxford: Oxford Medical Publications.

Dubois MY. 2005. The birth of an ethics charter for pain medicine. Pain Med, 6:201-2.

European Pain in Cancer Survey, European Association of Palliative Care. 2007. Half of European cancer patients have moderate to severe pain: one in five patients does not receive treatment. J Pain Palliat Care Pharmacother, 21:51-3.

European Society for Medical Oncology (ESMO) Guidelines Task Force. 2005. ESMO Minimum Clinical Recommendations for the management of cancer pain. Ann Oncol, 6(Suppl 1).

ESMO Guidelines Task Force. 2007. Management of cancer pain: ESMO Clinical Recommendations. Ann Oncol, 18(Suppl 2).

Furlan AD, Sandoval JA, Mailis-Gagnon A, et al. 2006. Opioids for chronic noncancer pain: a meta-analysis of effectiveness and side effects CMAJ, 174: 1589-94.

Glare PA, Walsh TD. 1993. Dose-ranging study of oxycodone for chronic pain in advanced cancer. J Clin Oncol, 11:973-8.

Goudas LC, Bloch R, Gialeli-Goudas M, et al. 2005. The epidemiology of cancer pain. Cancer Invest, 23:182-90.

Italian Department of Health. 2007. [online]. Accessed 6 August 2007. URL: www.Redazione-Ministerosalute.it.

Italian Medical Oncology Association (AIOM). 2007. [online]. Accessed 6 August 2007. URL: www.fondazioneaiom.it.

Kehlet H, Dahl JB. 2003. Anaesthesia, surgery, and challenges in postoperative recovery. Lancet, 362:1921-8.

Mameli M, Maurizi P, Morino P, et al. 2006. Adeguata titolazione per un utilizzo di ossicodone $\mathrm{CR}$ ad alte dosi: chiave di successo nella risoluzione del dolore? Risultati del progetto DADO (Definizione Alti Dosaggi Ossicodone). Clinical Intervent Aging, Companion Series, I:1.

Napp Pharmaceuticals. 2007. Summary of Product Characteristics: OxyContin tablets, 5 June 2007.

Portenoy RK, Farrar JT, Backonja MM, et al. 2007. Long-term use of controlled-release oxycodone for noncancer pain: results of a 3-year registry study. Clin J Pain, 23:287-99.

Reder R, Shi M. 1993. Safety and efficacy of controlled release oral oxycodone: doses greater than $80 \mathrm{mg}$ per day [poster]. 7th World Congress on Pain, Paris, France; August 22-27 1993.

Riley J, Ross JR, Rutter D, et al. 2006. No pain relief from morphine? Individual variation in sensitivity to morphine and the need to switch to an alternative opioid in cancer patients. Support Care Cancer, 14:56-64.

Staahl C, Dimcevski G, Andersen SD, et al. 2007. Differential effect of opioids in patients with chronic pancreatitis: an experimental pain study. Scand J Gastroenterol, 42:383-90.

World Health Organization (WHO). 1996. Cancer pain relief with a guide to opioid availability. Geneva: World Health Organization.

Yang Y, Chengyuan W. 2007. Guidelines on the basic outcome data from International Association for the Study of Pain. Clin J Pain, 23:549. 
